\title{
A User-Driven Process for Mining Association Rules
}

\author{
Pascale Kuntz, Fabrice Guillet, Rémi Lehn, and Henri Briand \\ IRIN - IRESTE/Ecole polytechnique de l'université de Nantes \\ La Chantrerie BP 6060144306 Nantes CEDEX 3 - France \\ $\{$ kuntz, guillet, lehn, briand\}@irin.univ-nantes.fr
}

\begin{abstract}
This paper describes the components of a human-centered process for discovering association rules where the user is considered as a heuristic which drives the mining algorithms via a well-adapted interface. In this approach, inspired by experimental works on behaviors during a discovery stage, the rule extraction is dynamic : at each step, the user can focus on a subset of potentially interesting items and launch an algorithm for extracting the relevant associated rules according to statistical measures. The discovered rules are represented by a graph updated at each step, and the mining algorithm is an adaptation of the well-known A Priori algorithm where rules are computed locally. Experimental results on a real corpus built from marketing data illustrate the different steps of this process.
\end{abstract}

\section{Introduction}

As well-kwown by data analysts (e.g. [3]), appropriate visualization supports often give an insight into data that would be more difficult to get from looking at long listings of output, and consequently makes the user-interaction easier. During the upstream steps comprising the KDD process i.e. data mining and post-treatment where discovered knowledge is evaluated, visualization tools are targeted to several major objectives [16. They allow to intelligibly describe basic relationships -often statistical- between data, and they contribute to the exploratory analysis by facilitating comparisons with more sophisticated models associated with hypotheses on data. Hence, numerous models previously developed in statistical analysis or machine learning have been integrated in software. The rapid development of new technologies such as virtual reality further stimulates the investigation of new paradigms of data representation (e.g. 6]). All of these tools make data and result handling easier, but most of the existing systems reported in the literature still let the decision-makers cope on their own with various diagrams. They do not explicitly take into account the cognitive capacities, in particular the domain knowledge, of each user. Unfortunately, the expression of the decision-maker's goals still remains a sensitive problem in KDD, and the current complexity of the knowledge representation systems limits their transfer into a KDD process.

D.A. Zighed, J. Komorowski, and J. Żytkow (Eds.): PKDD 2000, LNAI 1910, pp. 483-489, 2000 (C) Springer-Verlag Berlin Heidelberg 2000 
A pragmatic approach to get round these difficulties is to include the analyst into the discovery loop : the decision-maker is considered as a heuristic which drives the mining algorithms via a well-adapted interface. Such a human-centered approach has known an increasing development in Decision Aid during the last decade -from decision making in selection tasks to manufacturing and process control 17, and some models start being developed in KDD [5].

In this paper, we are concerned with discovery of association rules $X \rightarrow Y$ where $X$ and $Y$ are disjoint itemsets describing objects in a database. We describe a human-centered mining process which extracts rules bringing into play items that are a priori relevant for the user. Our approach has been inspired by experimental works on user behaviors during a discovery stage [2], [18, and by some cognitive mechanisms studied in decision models [19]. These works show on not only that, due to his short-term memory capacity, a decision maker manipulates a small amount of information at each step, but also that the processed information that leads to decisions has to be large enough for individual or social justification. Hence, in our case, we develop a dynamic model where the user can focus on a small subset of items at each step and can globally extract a significant set of association rules represented by a graphical model.

\section{Rule Representation}

We consider a large set of $n$ objects $O=\left\{o_{1}, \ldots, o_{n}\right\}$ described by the items $I=\left\{i_{1}, \ldots, i_{p}\right\}$. Although our approach may be generalized to more complex data, here we restrict ourselves to binary items. In this case, each object $o_{i}$ is described by a subset $X_{i}$ of $I: X_{i}=\left\{i_{1}, i_{4}, i_{5}\right\}$ means that only items $i_{1}, i_{4}$ and $i_{5}$ are present for $o_{i}$,

\section{Graphical Models for Implications}

Amongst visualization techniques, graphs often have a privileged place, in particular for rule relationship representation. They can be used at the same time as theoretical models and as representation tools[7].

For purely logical rules, i.e. without any counter-examples, Galois lattices [4] have known a renewed interest in the last decade in Combinatorial Data Analysis for searching implications in binary data [8]. Let us recall that each node of the lattice is a pair, composed of a subset of $O$ and a subset of $I$ and that the set of pairs is ordered by the standard set inclusion relationship applied to $O$ and $I$. This partially ordered set can be represented by a Hasse diagram but, unfortunately, determining a Galois lattice along with its Hasse diagram is a computationally difficult problem [15], and the representation become inextricable for numerous items.

We here develop a simpler model which does not take into account the Galois connection between subsets of $O$ and $I$, and whose incrementality is directed by the user. A rule network is represented by a directed acyclic graph $G=(V, A)$ where each vertex of $V$ is a potentialy interesting itemset, and each arc of $A$ represents a significant implication between two itemsets. Formally, a vertex is a subset $X$ of $I$, and an arc exists between two vertices $X$ and $X^{\prime}$ if the rule 
$X \rightarrow X^{\prime} \backslash X$ is valid according to statistical measures described below. For instance, if $X=\left\{i_{4}, i_{7}\right\}$ and $X^{\prime}=\left\{i_{4}, i_{7}, i_{9}\right\}$, an arc between $X$ and $X^{\prime}$ is associated with the rule $\left[i_{4} \wedge i_{7}\right] \rightarrow i_{9}$.

Statistical Measures of Rule Quality

Quality of discovered rules is measured here by statistical measures. Intuitively, a rule $X \rightarrow Y$ is statistically significant if it is covered by a large number of objects and refuted by few. Many coefficients have been presented in the literature to quantify these features, but from experimental comparisons [11] and [13], we retain three complementary measures :

1) The support $\rho(X \rightarrow Y)$ is the proportion of transactions which satisfy the rule. Let $g(X)$ be the maximal transaction subset covered by the itemset $X$. Then , $\rho(X \rightarrow Y)$ is the ratio $|g(X \cup Y)| /|O|$.

2) The conditional probability -or confidence- $\pi(X \rightarrow Y)=|g(X \cup Y)| /|g(X)|$.

3) The intensity of implication $\varphi(X \rightarrow Y)$ measures the "surprise" of having few counter-examples for the $X \rightarrow Y$ rule as compared with a random law. This measure was introduced by Gras 12 to improve the evaluation of rule confidence. The basic idea of intensity of implication is to compare the number of counter-examples $N(g(X), g(\bar{Y}))$ of the rule $X \rightarrow Y$ with the expected number $N\left(U, \overline{U^{\prime}}\right)$ where $U$ and $U^{\prime}$ are two randomly selected subsets of $O$ considered to be equals to respectively $|g(X)|$ and $|g(Y)|$. Then, the rule is surprising when the probability $P=\operatorname{Pr}\left(N(g(X), g(\bar{Y})) \leq N\left(U, \overline{U^{\prime}}\right)\right)$ is small. The intensity of implication is defined by $\varphi(X \rightarrow Y)=1-P$.

\section{Interactive Visualization of the Model}

The successive mining steps of the user are modeled by a series of graphs $G_{t}=$ $\left(V_{t}, A_{t}\right)$ with the vertex set $V_{t}$ and the arc set $A_{t}$ defined in section 2 The graph $G_{t}$ at step $t$ is deduced from the graph $G_{t-1}$ at step $t-1$ by vertex and arc insertions.

Initially, the graph $G_{0}$ only contains vertices representing itemsets $X$ of cardinality one associated with a support $|g(X)| /|O|$ fixed by the user. At each step $t$, the user selects a vertex and the significant associated rules are automatically generated by the algorithm described below. Then, a new graph, $G_{t+1}$, is drawn to update the knowledge. By moving sliders, he can also, at each $t$, modify the threshold values of the quality measures of the rules. This operation entails the deletion or the insertion of some arcs.

To make the interactivity easy, the different graph layouts must be intelligible. Common adopted readability criteria attempt to characterise readability by the means of general combinatorial optimisation goals such as minimizing arc crosses and minimizing the sum of the arcs' lengths to avoid long lines which can create confusion. Moreover, a drawing convention which precise vertex and arc characteristics must be specified. We consider here polyline drawings with vertices arranged in vertical layers. 
Each layer is associated with a degree of precision in the knowledge state : layers at the left correspond to general characteristics described by few attributes whereas layers at the right are more specific.

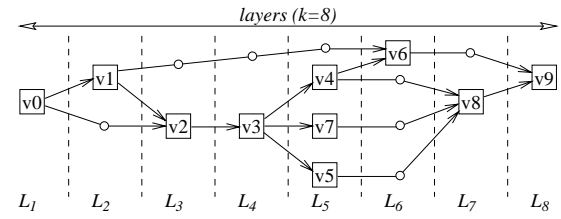

When $t$ is fixed, this polyline drawing problem is known to be NP-complete 9. Different efficient algorithms have been proposed, but they do not take into account the user interactivity. If a modification is performed, the algorithm runs again and produces a new drawing which may be completely different from the previous one. This requires the user to continually re-analyse the entire drawing, and important changes between consecutive layouts highly disturb the interpretation task. Hence, our interactive mining process requires drawing methods which preserve the "user's mental map" as much as possible. The formalization of these cognitive constraints and their treatment remain a difficult question which is far beyond the scope of this paper and we refer to 14 for the description of an efficient genetic algorithm we developed for this problem.

\section{Extraction Algorithm}

The rule computation can be split up into two separated stages. Initially, large itemsets, i.e. itemsets $X$ with a support $|g(X)| /|O|$ larger than a given threshold minSup and a cardinality bounded by a constant maxDepth, are computed with the same procedure as in the first stage of the well-known A Priori algorithm 11. There, at each step $t$, once a vertex has been selected by the user on $G_{t}$, the associated rules are extracted with a particular local procedure.

By selecting an itemset $X$, the user can trigger two different algorithms according to his own goals :

A1 for computing new "local rules" of type $X \rightarrow\{i\}$ with item $i \in I \backslash X$, which are more specific. This case corresponds to a "forward chaining" which highlights conclusions that are infered from the current state.

A2 for computing more general rules of type $X \backslash\{i\} \rightarrow\{i\}$ with item $i \in X$. This case corresponds to a "backward chaining" which highlights premises that allow to conclude on the current state.

Let us denote by $L_{k}$ the set of large itemsets of cardinality $k$, and by $c_{X}=(X,|g(X)|)$ the description of each large itemset $X$. Figure 1 describes the algorithm $\mathbf{A} 1$, triggered at step $t$ by the user selection of item $X$. We suppose that $X \in L_{k}$. The algorithm A1 first selects into $L_{k+1}$, the subset $S_{X}$ of itemsets including $X$. Secondly the set $R_{X}$ of the more specific rules is deduced from $S_{X}: R_{X}$ is the set of rules $X \rightarrow Y$ so that $X \backslash Y \in S_{X}$.

In this case, the number of computed rules is bounded by the number of items $|I|$ and consequently, the algorithm $\mathbf{A} 1$ has a polynomial complexity in $O(|I|)$.

The algorithm A2 follows a similar principle : the set $R_{X}^{\prime}$ of the more general rules is computed by selecting into $L_{k-1}$ the subset $S_{X}^{\prime}$ of itemsets included in $X$. The complexity is in $O(|I|)$. 
Therefore, when the mining process is composed of $T$ steps, the rule discovery loop has a total complexity in $O(T .|I|)$ which has the advantage of being polynomial in number of items.

\section{Illustration}

We present experimental results on a real database which contains the results of a marketing survey on domestic appliances. Questions were mainly focused on ownership, satisfaction, future purchase projects concerning 23 appliance categories. The database comprises 5074 answers associated with 115 boolean attributes as for instance $r x$ which means "the consumer whishes to replace the machine coded by $x "$.

This survey aims at optimizing the marketing operations for these categories : determining what kind of equipment to propose to consumers owning another category, ... Let us notice that, in this case, no consumer category is defined $a$ priori and that the search is non-supervised.

Figures 2 a., 2 b. and 2 cr. show different steps of the mining process of a user. Figure 2a. corresponds to a common initial situation. A single item i.e. a large itemset of $L_{1}$ is selected and the associated rules are extracted by the algorithm $\mathrm{A} 1$. Here the item h11 means "the consumer is happy with his washing machine" and a discovered rules is for instance : $\mathrm{h} 11 \rightarrow \mathrm{h} 01 \wedge \mathrm{h} 11$ which means that " $a$ consumer who is satisfied by his washing machine is also satisfied with his TV set". All these rules have a confidence and a intensity of implication value greater or equal than 0.9

Figure $2 \mathrm{~b}$. describes the discovered graph after three successive requests concerning consumers satisfied with their washing machine: a "backward chaining" on $011 \wedge \mathrm{h} 11$ followed by a "forward chaining" on 011 and $001 \wedge 011 \wedge 015$ ("ownership of TV set, video tape recorder, washing machine and refrigerator").

In order to discover more general informations concerning the notion of ownership of common appliances, the user engaged backward chaining (algorithm

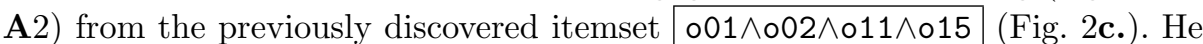
tries to determine wether the ownership of some of these appliances entails the ownership of the others. The graph shows that, for the chosen rule quality thresholds, the ownership of a video tape recorder and a refrigerator entails the ownership of the whole set of these usual appliances while the ownership of a TV set or a washing machine does not (see the first step in backward inference from $001 \wedge 002 \wedge 011 \wedge 015$ on Fig. 2r.).

Integrating the user in the KDD process as a heuristic in the rule mining task not only dramatically decreases the rule search space, but also allows the user to focus on potentially meaningful knowledge. It also opens new research perspectives in improving the intelligibility of the knowledge representation. We currently investigate on two distinct directions to enhance the readibility of the rule networks. We aim at eliminating the "redundant knowledge" by concealing the rules that the user can easily infer himself and we carry on perfecting a highly-interactive rule interface. 
- A1 : More specific rules :

$X:=C_{X}$.itemset; $k:=|X|$

// Clicked itemset and its size

$S:=\left\{C_{Y} \in L_{k+1} \mid X \subset C_{Y}\right.$.itemset $\}$

// large $k+1$ itemsets access

$R_{X}:=\emptyset$

For each $C_{Y} \in S$

$Y:=C_{Y}$.itemset

r.rule $:=X \rightarrow Y \backslash X$

$r$.support $:=C_{Y}$.support

$/ /$ the set of more specific rules $/ /|S| \leq\left|I_{u}\right|-|X|<<\left|L_{k+1}\right|$

$r$.confidence $:=C_{Y}$.support / $C_{X}$.support

// a new rule ( $Y \backslash X \mid=1)$

$C_{Y \backslash X}:=\left\{c \in L_{1} \mid c\right.$. itemset $\left.=Y \backslash X\right\}$

$r$.intensity $:=\varphi\left(\left|I_{u}\right|, C_{X} \cdot\right.$ support,

$R_{X}:=R_{X} \cup\{r\}$

$/ /$ its quality indices

\section{End for each}

Fig. 1. Algorithm A1 for local rule computation. (minConf and minInt are respectively the confidence threshold and the intensity of implication threshold.)

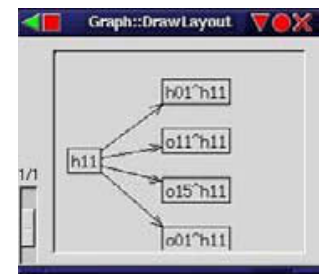

a. h11 selection

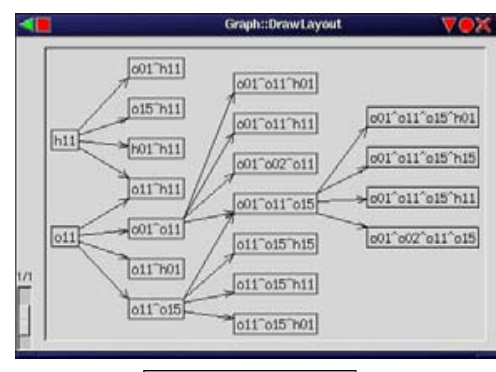

b. $001 \wedge 011 \wedge 015$ selection.

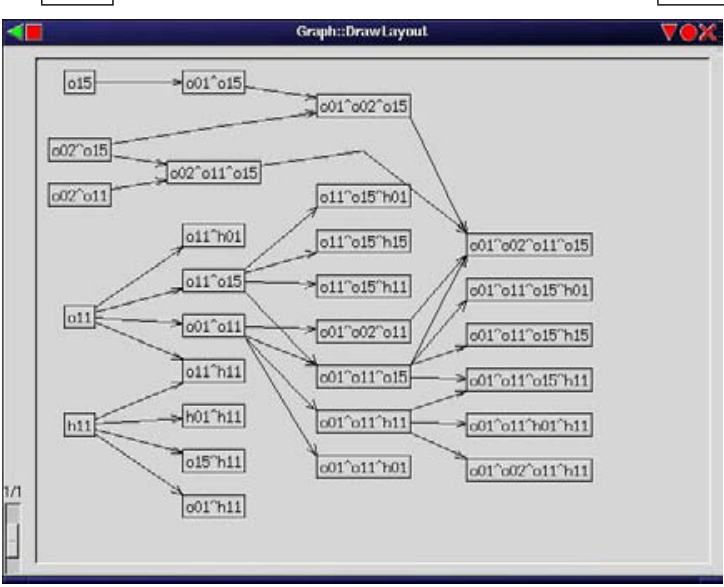

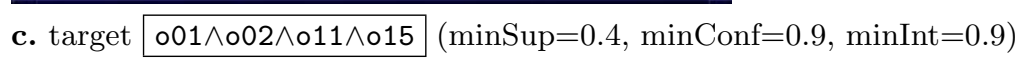

Fig. 2. Discovered rules following different vertex selection. 


\section{References}

1. R. Agrawal, H. Mannila, R. Srikant, H. Toivonen, and A.-I. Verkamo. Fast discovery of association rules. In Fayyad et al. [10], pages 307-328.

2. I. Bandhari. Attribute focussing: machine-assisted knowledge discovery applied to software production process control. Knowledge Acquisition, 6:271-294, 1994.

3. J. Bertin. Graphics and Graphic Information Processing. Berlin: Springer, 1981.

4. G. Birkoff. Lattice Theory. A.M.S, 1967.

5. J.R. Brachman and T. Anand. The process of knowledge discovery in databases: a human-centered approach. In Fayyad et al. [10], pages 37-58.

6. C. Brunk, J. Kelly, and R. Kohavi. Mineset: An integrated system for data mining. In Proc. of the 3rd Int. Conf on Knowledge Discovery and Data Mining, pages 135139. AAAI Press, 1997.

7. W. Buntine. Graphical models for discovering knowledge. In Fayyad et al. 10, pages $59-82$.

8. V. Duquenne. Latticial structures in data analysis. Theoretical Computer Science, 217(2):407-436, 1999.

9. P. Eades and N. Wormald. Edge crossings in drawings of bipartite graphs. Algorithmica, 11:379-403, 1994.

10. U.M. Fayyad, G. Piatetsky-Sapiro, and P. Smyth. From data mining to knowledge discovery. In U.M. Fayyad, G. Piatetsky-Sapiro, and P. Smyth, editors, Advances in Knowledge Discovery and Data Mining, pages 2-34. AAAI Press, 1996.

11. L. Fleury, H. Briand, J. Philippé, and C. Djeraba. Rule evaluation for knowledge discovery in databases. In Proc. of the $6^{\text {th }}$ Int. Conf. on Database and Expert System App., pages 405-414, 1995.

12. R. Gras, H. Briand, and P. Peter. Structuration sets with implication intensity. In Proc. of the Int. Conf. on Ordinal and Symbolic Data Analysis, pages 147-156, 1996.

13. S. Guillaume, F. Guillet, and J. Philipp . Improving the discovery of association rules with intensity of implication. In Proc. of the Second European Symposium on Principles of Data Mining and Knowledge Discovery, pages 318-327. Lect. Notes in Comp. Sc., Springer-Verlag, 1998.

14. F. Guillet, P. Kuntz, and R. Lehn. A genetic algorithm for visualizing networks of association rules. In Proc. of the 12th Int. Conf. on Industrial and Engineering App. AI and Expert Sys., pages 145-154. Lect. Notes in Comp. Sc., Springer-Verlag, 1999.

15. A. Gu noche. Building the galois lattice of a binary relation (in french). Math matiques, Informatique et Sciences Humaines, 109:41-53, 1990.

16. D.A. Keim and H.-P. Kriegel. Visualization techniques for mining large databases: A comparison. Trans. on Knowledge and Data Engineering, Special Issue on Data Mining, 8(6):923-938, 1996.

17. P. Lenca, editor. Proc. of the 10th Mini Euro Conf.: Human Centered Processes. Brest: Ecole Nationale Sup rieure des T l communications de Bretagne, 1999.

18. J. Philippé, T. Teusan, S. Baquedano, and C. Bourcier. The implicative analysis in a knowledge extraction context for perfecting decision aid systems in behavioral analysis (in french). In Actes des journ es sur La fouille dans les donn es par l'analyse implicative (to appear), 2000.

19. O. Svenson. Decision rules and information processing in decision making. In Human decision making. Bodafors: Bodaforlaget Doxa, 1983. 